\title{
Generation of Induced Pluripotent Stem (iPS) Cells by Nuclear Reprogramming
}

\author{
Dilip Dey and Gregory R. D. Evans \\ Aesthetic and Plastic Surgery Institute, University of California, Irvine, Orange, CA 92868, USA \\ Correspondence should be addressed to Dilip Dey, ddey@uci.edu \\ Received 16 March 2011; Revised 18 June 2011; Accepted 22 June 2011 \\ Academic Editor: Mark G. Carter
}

Copyright ( 2011 D. Dey and G. R. D. Evans. This is an open access article distributed under the Creative Commons Attribution License, which permits unrestricted use, distribution, and reproduction in any medium, provided the original work is properly cited.

During embryonic development pluripotency is progressively lost irreversibly by cell division, differentiation, migration, and organ formation. Terminally differentiated cells do not generate other kinds of cells. Pluripotent stem cells are a great source of varying cell types that are used for tissue regeneration or repair of damaged tissue. The pluripotent stem cells can be derived from inner cell mass of blastocyst but its application is limited due to ethical concerns. The recent discovery of iPS with defined reprogramming factors has initiated a flurry of works on stem cell in various laboratories. The pluripotent cells can be derived from various differentiated adult cells as well as from adult stem cells by nuclear reprogramming, somatic cell nuclear transfer and so forth. In this review article, different aspects of nuclear reprogramming are discussed.

\section{Introduction}

Adult human beings cannot regenerate organs as the regeneration has silenced during evolution to protect from tumorigenesis. However, in human, part of the liver can regenerate. In lower animals, regeneration of organs or part of organs are very common. Song birds' brain tissue that controls the singing of birds dies after every breeding season and that lost neuron is again are replaced at the advent of next breeding season [1]. Reptiles like lizards lose their tail to deceive predators and the lost tail regenerates. Terminal differentiated cells like fibroblasts are thought to be unable to convert to other cell types. However, cloning experiments in amphibians and later in mammals proved that indeed they have the potential to reprogram and generate other cell types $[2,3]$.

Human adult neurons, heart tissues never thought to regenerate but in the late 90's it was found that in occasional cases human adult neurons divide [4].

It is now established that terminal differentiation of cells is a reversible process that has generated a lot of interest in reversion of cell differentiation and reprogramming to iPS. Stem cells can divide unlimitedly and can give rise to its own and different kinds of adult cells. It holds the promise for the treatment of several neurological and other debilitating diseases like Parkinson, Alzheimers, ischemic heart failure, diabetes mellitus, Huntington disease, and sickle cell anemia [5].

\section{Sources of Pluripotent Cells}

Several methods of pluripotent cell derivation exist. (a) Embryonic stem (ES) cells can be harvested directly from the inner cell mass (ICM) of preimplantation blastocyst. (b) Somatic cell nuclear transfer (SCNT) is a therapeutic technique where adult somatic nuclei are microinjected into enucleated eggs. The egg, now containing the nucleus of a somatic cell, is stimulated with a shock and the somatic cell nucleus is reprogrammed by the host egg cell and forms a blastocyst. (c) Cellular hybridization where pluripotent hybrid cells are formed via somatic cell fusion with an ES cell. (d) Induced pluripotent stem (iPS) cells are developed from-patient-specific somatic cell reprogrammed to an ES cell-like state. For practical purpose, hES cells have faced difficulties because of ethical issues, potential immuno-incompatibility, and an increase of MHC molecule 
TABle 1: Nuclear reprogramming: various cell types that can be reprogrammed are shown in Table 1. During reprogramming several cell markers for pluripotency or differentiation are upregulated or downregulated.

\begin{tabular}{ll}
\hline Phenomena & Factors \\
\hline $\begin{array}{l}\text { Starting cell } \\
\text { types }\end{array}$ & $\begin{array}{l}\text { Keratinocytes, t-cells, fibroblasts, Adipose-derived stem cells (ADSCs), mesenchymal stem cells (MSCs), dental pulp } \\
\text { stem cells, germ line stem cells, neural stem cells, cord blood stem cells, hair follicle, retina, skeletal muscle. }\end{array}$ \\
\hline & (a) Embryonic development-upregulated (Oct4, Sox2, Nanog, Lin-28, NR5A2, TBX3, STAT3 and ZIC3). \\
& $\begin{array}{l}\text { Downregulation of differentiation specific genes (PAX6, ATBF1 and SUZ12). } \\
\text { (b) Proliferation-upregulated (Cyclin D1, c-Myc, KLF4, Rem2). } \\
\text { Factors }\end{array}$ \\
Upregulated/ & Downregulated (p53, p21, p16 ${ }^{\text {INK4A }) .}$ \\
Downregulated & (c) Epigenetic-downregulated (DNMT1, HDAC, Histone demethylase). \\
& (d) Signalling pathway-downregulated (TGF $\beta$, Wnt/ $\beta$ catenin, PI3/AKT). \\
& (e) Chromatin-opened (by SWI/SNF). \\
& (f) Miscelleneous-to express (Vitamin C, hypoxia, TERT, E-cadherin). \\
\hline
\end{tabular}

expression during differentiation [6]. SCNT for humans are difficult to achieve and mired with ethical issues related to egg destruction. Similarly, the utilization of ES cells has been difficult due to ethical reasons. As an alternative to ES, the establishment of iPS cells has raised more interesting potential as they are created from adult cells [7]. In his seminal work on iPS, Yamanaka et al. used 4 reprogramming factors (they are also transcription factors) such as Oct3/4, Sox2, Nanog, and c-Myc to reprogram mouse somatic cells. Later, reprogramming factors (RFs) delivery were done by retroviral and lentiviral vectors and other nonviral delivery systems (Table 1). The minicircle (MC) delivery system is designed to deliver TFs episomally to avoid the unintended consequences of viral use in the clinical setting [8]. Recently, however, mRNA with 4 reprogramming factors added directly to the cell demonstrated a high efficiency of reprogramming of adult skin cells [9]. Reprogramming efficiency has also improved with the use of various chemical compounds and growth factors (such as Wnt and TGF- $\beta$ signaling pathways) in addition to 4 reprogramming factors (Figure 1).

\section{ES and iPS Cells}

ES and iPS cells have the ability to produce almost any types of adult cells. However, use of ES cells for clinical purpose is controversial. Additionally, cells derived from ES cells can be rejected by the host immune system as they are autogenously derived. To overcome the ethical and immune rejection issues, iPS cells are developed from adult differentiated cells by reprogramming factors. This iPS, which is morphologically and characteristically close to ES, can be differentiated to various types of tissues and replenish host tissue loss in neurodegenerative and other diseases. Direct reprogramming of cells by iPS is more convenient and reliable than generation of pluripotent cells from ICM of blastocyst [10]. However, different studies have shown that iPS cells are not as superior as ES cells.

Before starting any reprogramming, we need to understand genetic and epigenetic changes at the molecular level that causes reprogramming from a differentiated cell to a pluripotent one. There is a gulf between lab bench success on
iPS and clinical application of this technology as still characterization is taking place. For the safety concerns need to be addressed before any clinical consideration. The bottlenecks of iPS are inadequate cell number, immune rejection, and tumor (teratoma) formation. All the pluripotent cells (hESC, iPS, and NTSC) are potentially tumorigenic. More research is needed to overcome this problem [11].

\section{Cell Types for Reprogramming}

Before starting any reprogramming, information about the plasticity of the starting cell is helpful as cellular reprogramming is an interplay between plasticity and environmental factors like epigenetic modifications. Both adult stem cells and terminally differentiated cells can be reprogrammed but efficiency, time period, and extent of reprogramming varies among the starting cells. The derivation of functional neurons, cardiomyocytes, pancreatic islet cells, hepatocytes, and retinal cells prove that it is possible to derive cells from many sources of tissues by reprogramming, thus facilitating the various treatments. The starting cells should not be necessarily from the organ that will require repair. As an example, regeneration of insulin-producing cells can be done from primary source (in this case pancreatic $\beta$ cells), insulin-producing cells can be also derived from other alternative sources like ES, adult stem cell, mesenchymal and hematopoietic stem cells. This was also achieved by various means like nuclear reprogramming and transdifferentiation. Bone marrow tissues are a source of adult mesenchymal stem cells but are collected by more invasive and painful surgery. Adipose-derived stem cell (ADSC) is the most abundant and can be easily isolated from the patient by local anesthesia. Interestingly fat tissues contain about 100 -fold adult stem cells more than bone marrow making it an attractive source for adult stem cells. Human ADSCs are a heterogeneous group of multipotent progenitor cells and it has certain advantages; they can be collected autogenously in high numbers $(100 \mathrm{~mL}$ of human adipose tissue yields about $1 \times$ $10^{6}$ cells) with minimal morbidity [12]. Additionally, ADSCs express 3-4 times endogenous Klf4 compared to human ES cells and 1.3 times higher expression of c-Myc both are reprogramming agents. Reprogramming of ADSCs to 


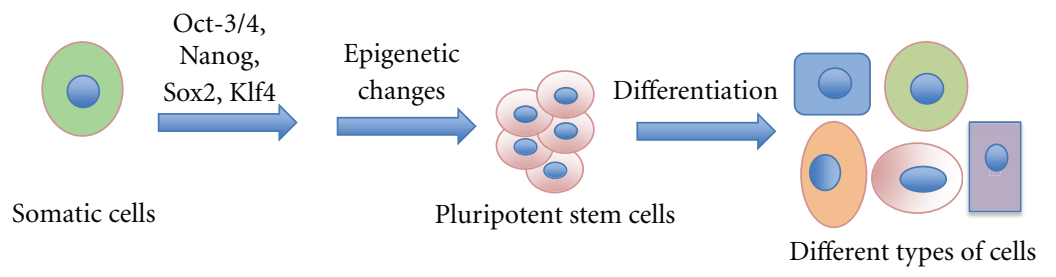

(a)

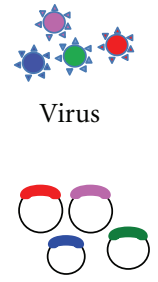

Plasmid

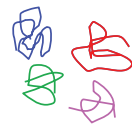

Protein

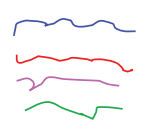

mRNA

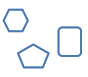

Small chemicals

(b)

FIGURE 1: A simplified version of reprogramming of adult cells to pluripotent cells by 4 factors. (a) Any adult source of cells like epithelial, muscle, fibroblast, and adipose cells is isolated and cultured in appropriate medium. Then cells are transfected, nucleofected, or transduced by 4 reprogramming factors. These factors may be Oct3/4, Sox2, Nanog, and Klf4/c-Myc. The reprogramming paths are not well understood yet, requiring expression of several genes as well as epigenetic modification of the genome. (b) Reprogramming factors (transcription factor genes) can be delivered by various methods. In case of viral gene delivery, genes are integrated with the host chromosome randomly. Methods of gene deliveries like plasmids, mRNAs, proteins, genes are expressed transiently and diminished the expression with the concomitant increase in expression of pluripotent markers.

iPS and subsequent neuronal differentiation is an attractive alternative to hES. ADSCs can be induced to differentiate into osteogenic [13], chondrogenic [14], adipogenic [15], hepatic [15], cardiogenic [16], neurogenic [17], islet-like insulin secreting cells [18], and hematogenic (mouse) lineages [19].

\section{The Reprogramming Vectors}

5.1. Reprogramming by Retroviral Vectors. The reprogramming with gene delivery system that remains as episome (like adenovirus, mRNA, and minicircle) has great advantage over the integration of genes into the host chromosome (hantavirus, retrovirus). The reprogramming factors are expressed transiently and then degraded or diminished. This primes and drives the host cell to a choreography of downstream reactions (pathways are not yet well understood) that ultimately convert the cell to pluripotency. For DNA-based (viral vector) reprogramming, 4 proteins trigger reprogramming but DNA remains altered due to viral integration into the chromosome. Nevertheless, the efficiency of retroviral reprogramming is at very low frequency (less than $0.1 \%$ ). In the original reprogramming method a retrovirus (MMLV) was used; the RNA virus carried the transgene and reversetranscribed inside the host cell. This DNA was integrated into the host chromosome and was a constant source of transgene protein. The retroviral promoter was inactivated probably by histone modification (methylation) [20]. Reprogramming of adult cells by viral delivery of 4 reprogramming factors (Oct4, Sox2, Nanog, and c-Myc) have been used successfully for iPS generation. Takahashi et al. first developed mouse iPS and one year later established human iPS [7, 21]. These iPS cells are supposed to be equivalent or comparable to ES cells in morphology, gene expression, and epigenetic status and can give rise to 3-germ layers. There are some disadvantages of retroviral vectors. It integrates randomly in host chromosome making leaky expression and transforms the cell into tumor. Besides, the random integration also makes cells heterogeneous and iPS cells should be screened for various clones (efficacies and safety). Using c-myc as an agent of reprogramming factors makes cell more tumorigenic as the role of myc has been well documented in connection with tumor. Myc is an immediate early gene and reactivation of $\mathrm{c}-\mathrm{Myc}$ gave rise to transgene-derived tumor formation in chimeric mice [22]. Chimeric mice produced with cmyc-free iPS did not develop any tumor until 6-month observation period compared to control mice. Besides cMyc, various tumors also express OCT3/4, SOX2, and KLF4 the other 3 reprogramming factors. Overexpression of these 3 factor-derived iPS causes tumor formation. Hopefully, there are some refinements to the above procedures. Some labs have minimized the number of integration into the host genome by putting all reprogramming factors in one vector. This is technically/practically accomplished by putting IRES sequence between reprogramming genes or a self -cleavage 2A peptide sequence. This way iPS cells are produced with only single insertion in the genome [23].

5.2. Reprogramming by Nonviral Vectors. Until now, there have been several nonviral vectors designed for TFs delivery. A partial list of them is shown in Table 2. Minicircle (MC) vectors have been developed by Dr. Joseph Wu's team at 
TABLE 2: Nuclear reprogramming factor delivery. List of reprogramming factors delivery by various methods shown in Table 2.

\begin{tabular}{ll}
\hline Mode of delivery & Vectors \\
\hline Viral delivery & MMLV retrovirus, lentivirus, adenovirus, hantavirus, transposon, retrotransposon. \\
& PiggyBack, plasmid, minicircle, synthetic mRNA, IVT mRNA, protein, cell fusion (ES + adult), \\
Episomal delivery & cell fusion (tumor + adult), protein delivery by gag protein, \\
Small molecule (chemical) & ALK5 inhibitor, GSK3 inhibitor, and MEK inhibitor. \\
\hline
\end{tabular}

Stanford University [8]. Minicircles (MC) are circular nonviral DNA elements that are generated by an intramolecular (cis-) recombination from a parental plasmid (PP) pLGNSO (Lin28, GFP, Nanog, Sox2, and Oct3/4) mediated by $\phi \mathrm{C} 31$ integrase. Thus delivering only the minicircles to cells lengthens the expression of the transgene over traditional transient transfection of plasmids. For dividing cells, expression of the minicircles lasts up to 14 days. For nondividing cells, expressions of the minicircles drop slightly after the 1st week, but can continue the expression of transgenes for months. The beauty of minicircle is that MC possesses higher ectopic expression and less inactivation by cellular machineries. The plasmid contains a single cassette of 4 reprogramming factors and GFP coding sequences are linked by " $2 \mathrm{~A}$ " peptide sequences [24]. The minicircle when used to transfect the adult cell, gradually over time becomes ES type morphology without changing the global gene expression. Inside the cell, the MC is not integrated in the genome, but instead it is transcribed as a whole single long mRNA containing all the 5 genes ( 4 RFs \& GFP). This is translated as a single protein where individual proteins are processed by self-cleavage peptide $2 \mathrm{~A}$. In case of synthetic RNA-based reprogramming RNA is translated into protein in the cytoplasm and host DNA remains unaltered. So, synthetic RNA-based reprogramming is clean, safe, and fast and iPS cells are genetically identical to their source cells. The reprogramming process takes a little over 2 weeks with $4 \%$ of the cells being reprogrammed. This is about $100-$ fold more efficient than reprogramming by gene transfer technique [9]. They named it RNA-induced pluripotent stem (RiPS) cells. However, it is difficult to synthesize long mRNA chemically and researchers have not been able to make large mRNAs. Needless to say mRNA is prone to degradation and needs very rigorous quality control in the lab. The cells have a defense system against RNA virus infection. That is an old defense mechanism present in both plants and animals. The host cell perceives foreign RNA as a viral invasion and degrades RNA in small fragments by RISC machinery that is available inside the cells. This cell-mediated RNA degradation is known as RNA interference (RNAi) [25]. To circumvent that problem, Dr. Rossi's team chemically synthesized mRNA with some modified bases (nucleotides) at intervals so it is not recognized as an outsider RNA and protected from degradation. Yakubov et al. also confirmed reprogramming by mRNA of 4 TFs. However, they did not use synthetic RNA instead they used mRNA which was synthesized by in vitro transcription (IVT) from the DNAs of 4 genes. For continuous expression, they transfected a total of five times and established iPS that is alkaline phosphatase positive and expressed several pluripotent markers [26].
5.3. Model Reprogramming of Cells: hADSCs. Human ADSCs are a heterogeneous group of multipotent progenitor cells that can be collected autogenously in high numbers [27]. ADSCS are more abundant than bone marrow by a factor of 100. Nucleofector (Amaxa, Germany) is used for nucleofection with 4 reprogramming factors. Nucleofection is a poorly explained method where DNA materials are directly delivered to the nucleus. All cells are used for reprogramming are within early passage. Transfected hADSC cells are then seeded onto a Mitomycin C treated MEFs feeder layer or on Matrigel-coated tissue culture dishes (ES qualified, BD Biosciences). The feeder layer provides nutrition for the cells that are being reprogrammed. Mitomycin $\mathrm{C}$ or radiation treated cells are alive but they do not divide as the cell cycle is arrested by DNA damage response pathway. On days 4 and 6, hADSCs are transfected again with minicircles using lipofectamine type transfection reagent which is less toxic to the cells than nucleofection. Cells were switched to human ES cell culture medium prepared using DMEMF12+ 10\% knockout serum and $100 \mathrm{ng} / \mathrm{mL}$ of bFGF (FGF2 ). Colonies with morphologies similar to human ES cell colonies are expected to be visible in 3-4 weeks after transfection. Gradual loss of GFP expression due to dilution of minicircles followed by activation of endogenous Oct4 expression will be observed on successive cell proliferative cycles. During this reprogramming, cells divide and form round/circular compact colony of cells with a clear margin. In mouse cell reprogramming, leukemia inhibitory factor (LIF) is also used in the media to keep them in a pluripotent state while avoiding any differentiation.

The pluripotency of the iPS cells is determined by immunofluorescence for ES markers like Oct4, Sox2, Nanog, and so forth. For clinical applications, Southern blots are done to check any genomic integration of reprogramming genes. The isolated colonies look morphologically similar to hES. Immunostains for pluripotent markers and alkaline phosphatase will be positive. iPS cells typically express SSEA4, TRA-1-60, and Nanog but not SSEA1.

5.4. Some Less Used Reprogramming Methods. Various Gramnegative bacteria have type III secretion system (T3SS) that injects virulent proteins (regulated by a variable secretion signal sequence) into the eukaryotic cell cytoplasm. Bichsel et al. used Pseudomonas aeruginosa exotoxin ExoS to translocate Cre recombinase containing a Cre-NLS signal [28]. Upon infection, bacteria delivered Cre-NLS to undergo LoxP mediated chromosomal DNA recombination. This resulted in iPS and establishes the use of T3SS for the delivery of TFs in cellular reprogramming. Retroviruses assemble polymer of Gag protein which is proteolytically cleaved before entering 
the cell through receptor binding. This property of retrovirus was used to deliver the reprogramming proteins into the cells. Proteins were incorporated with nuclear localization signal (NLS) and tagged/attached to the retroviral protein. After cell entry, proteins were cleaved by retrovirus protease and the active TFs proteins were translocated inside the nucleus where it reprograms the cells. This expression is transient as proteins will be degraded after their intended job [29]. In routine iPS generation, germ-line transmission and live birth from iPS cells are produced by tetraploid complementation. It has been shown that addition of Tbx3 improves the quality of iPS cells. iPS cells derived with Tbx3 and Oct 4, SOX2, KLF4 are more successful in germ cell contribution [30].

Dermal papilloma (DP) cells are reprogrammed more efficiently than skin and embryonic fibroblasts. DP inherently express higher levels of SOX2 and c-Myc, so these DPs can be easily reprogrammed with only Oct4 and KLF4 [31]. Among the 4 reprogramming factors, Oct4 is a very important TF for iPS generation. Oct4 cannot be replaced with other TF whereas SOX2, KLF4 and c-Myc can be replaceable. However, $\mathrm{Ng}$ has shown that orphan (ligand is not known for the receptor) nuclear receptor Nr5a2 can be used for mouse somatic cell reprogramming with greater efficiency than Oct4. Genomewide expression analysis showed that Nr5a2 shares many target genes with SOX2 and KLF4 indicating they work together in concert [32]. The nuclear receptor superfamily has 48 genes. This nuclear receptor superfamily maintain various aspects of stem cell-like regulation of stemness, reprogramming of terminally differentiated cells [33]. An Oct-4 promoter-based reporter system has been developed in mouse and pig and is a useful tool for monitoring the differentiating status of porcine cells both in vivo and in vitro [34]. A recent work by Sugii et al. have improved the iPS efficiency with or without the feeder layer [35]. They also demonstrated that adipose stem cells (ASC) can be grown on ASC feeder layer instead of the MEF feeder layer. They reduced the iPS programming 1.5 and 2.5 weeks for mouse and human iPS, respectively. Terminally differentiated mature B lymphocyte has been reprogrammed with basic reprogramming factors plus the addition of C/EBPalpha [36]. Generally 4 reprogramming factors are added in equal ratio for iPS generation. However, increasing the concentration of OCT3/4 enhances reprogramming efficiency but increasing the concentration of SOX2, KLF4, and c-Myc reduces reprogramming efficiency [37]. The time required for reprogramming also varies among the starting cell types, source, and so forth. Mouse fibroblasts can be reprogrammed in 3 weeks whereas human fibroblasts with the same reprogramming factors require 4 weeks to reprogram. Interestingly, 4-factor reprogramming repressed cardiac differentiation due to prolonged expression of OCT4 and Fgf4. Contrary to that, 3-factor reprogramming (excluding c-Myc) enhances the expression of precardiac (CXCR4, Flk-1, and Mesp1/2) and cardiac-specific (Nkx2.5, Mef2c, and Myocardin) genes. The differentiation of cells showed continuous beating activity in a petri dish. So, reprogramming devoid of $\mathrm{c}-\mathrm{Myc} \mathrm{TF}$ has a tendency of preferentially generating cardiac tissues [38].
Human iPS induction was also done by adding several other factors like TBX3, mirna-291-3p, miR-294, and miR295. The adult newt can regenerate lens from pigmented epithelial cells (PECs) through dedifferentiation. It is like reprogramming iPS in vivo without any exogenous factors. The authors were interested in seeing which genes are being activated/expressed during the dedifferentiation procedure. They isolated mRNAs and constructed a cDNA library. Upon analysis, they found the expression of apoptosis and cancer-related genes and concluded that cancer and apoptosis-related genes expression may be a hallmark during dedifferentiation in newt [39]. Vitamin C enhances the generation of iPS in mouse and human somatic cells. It may play an auxilliary role to reverse senescence and promotes pre-iPS cells to complete programming of cells [40]. Lineage reprogramming has been an important tool for studying cell fate choice during differentiation. Several TFs that can drive cells from one lineage to another [41]. Induced expression of leukemic oncogene AML1-ETO in embryonic zebrafish reprogram hematopoietic progenitor cells from erythroid to myeloid.

5.5. Small Chemical Molecules. Small chemicals molecules that target enzymes of cell reprogramming pathways have been identified that control the cells fate like stem cell maintenance, reprogramming, and differentiation [42]. By using small synthetic chemical molecules a pluripotent state can be induced which is known as a chemically induced pluripotent cell (CiPSs) [43]. Li et al. has suggested that small molecules should not only be able to reprogram cells in vitro, but they can also be delivered into the body as conventional therapies to target a patient's own tissue for the treatment of degenerative diseases, injuries, and cancer (to target cancer stem cells) [44]. Cancer cells get resistant to chemotherapeutic drugs. It has been shown that reprogramming of GI cancer cells by the ectopic expression of TFs leads to reprogramming. The induced pluripotent cancer (iPC) cells were sensitized to chemotherapeutic drugs and also responded to differentiation-inducing treatment in short term cell culture [45]. Reprogramming of somatic cells is also possible by fusing ES cells with adult cells. In case of fusion-induced reprogramming, OCT4 reactivation starts within 1-2 days after ES cell and somatic cell fusion [46]. Reprogramming by fusion was achieved by the fusion of MEFs with mouse ES cells by using hemagglutinating virus of Japan envelope (HVJ-E). Microsatellite analysis of the derived stable cell line showed that they possess genes from both ES and MEF. The fused cells were tetraploid and positive for Alkaline Phosphatase (AP) and stem cell markers (OCT3/4, NANOG, SOX2) but not fibroblast cell marker (Colla1 and Colla2) [47]. Fusion between intestinal epithelial cells and macrophages in a cancer context results in nuclear reprogramming [48]. The structure of telomeric chromatin is dynamic and changes during cell transformation to cancer. Telomere is shortened in every cell cycle until it reaches a crisis stage where it goes to apoptosis [49]. Telomere expression is silenced in most adult somatic tissues except the adult stem cell compartments. 
During reprogramming telomeric chromatin is remodeled and telomeres are elongated by telomerase [50], although there are some heterogeneity during the reprogramming process with respect to telomere length [51].

5.6. Reprogramming by Transdifferentiation. Transdifferentiation is defined as the conversion of one cell type to another. Eberhard et al. used synthetic glucocorticoid dexamethasone and reprogrammed pancreatic cells to hepatocytes [52]. Cobaleda C dedifferentiated adult B cells into multipotent progenitor cells and afterward reprogrammed to alternative lineages $\mathrm{T}$ cells and macrophages [53]. Mak et al. used mammalian affinity purification and lentiviral expression (MAPLE) to deliver several protein complexes involved in transcription (RNA polymerase II associated factor, negative elongation factor, positive transcription elongation factor $b$, SWI/SNF complexes). They showed that TF KLF4 facilitates chromatin remodelling with the SWI/SNF complex [54].

The generation of animals by SCNT has shown that epigenome of differentiated cells can be reset to a pluripotent state. The nucleus from the somatic cell is inserted into an enucleated egg and can transform it into an embryonic stage (pluripotent). The cytoplasm of the egg is enough to reset the epigenome and DNA sequence remains unaltered. Compatibility of mitochondrial DNA haplotypes between donor cells and host oocytes improves reprogramming efficiency possibly by epigenetic modification. To increase the SCNT viability, treatment with chemicals that causes chromatin modification like Trichostatin-A (TSA) decreases expression of histone deacetylase (HDAC1 and HDAC2) and DNA methylation (DNMT3a and DNMT3b) while increases the expression of histone acetylation (P300 and CBP), pluripotency (OCT4 and NANOG) genes [55]. Histone deacetylase inhibitor valproic acid (VPA) enabled reprogramming of human fibroblasts with only Oct4 and SOX2 [56]. Wnt signalling results in inhibition of GSK-3 and stabilization of cytoplasmic $\beta$-catenin. Small molecule inhibitors of GSK3 can mimic the activation of wnt signalling and maintain the pluripotency of $m E S$ [57-59]. An immune system protein, activation-induced cytidine deaminase (AID) helps in reprogramming by DNA methylation. Thus important reprogramming genes Oct4 and nanog are induced. Li et al. has been able to reprogram somatic cells without the TF Sox2. TFs Oct4, and KLF4 alone can reprogram MEF when cultured with glycogen synthase kinase-3 (GSK-3) inhibitor CHIR 99021. They conclude that GSK-3 inhibitor may replace TF SOX2 in both mouse and human reprogramming. Additionally, CHIR 99021 in combination with parnate, TFs Oct4, and KLF4 are sufficient for reprogramming of human primary keratinocytes [60].

\section{In Vitro Differentiation of iPS Cells to Differentiated Cells}

Pluripotent iPS is like a hub from where differentiation to various lineage types is possible. For generation of mature neuron cells from iPS, cells are induced with culture media containing differentiation factors. Expression of differentiation marker is determined by the presence of $\alpha$-smooth muscle actin, $\alpha$-fetoprotein, and $\beta$-III tubulin. If viability of iPS cell is a problem, treatment of iPS cells with ROCK inhibitor Y-27632 before harvesting to increase their viability is possible. Recently cells can be induced to transdifferentiation by treatment with several transcription factors and transform cell from one lineage (fibroblast) to another (functional neuron) bypassing the conversion of pluripotent stem cell-like stage [61].

\section{Developing of Embryo, Pluripotent Stem Cells and Cancer Cells}

Embryo, pluripotent stem cells and cancer cells have some similarity in behavior. They are actively dividing and proliferated. Many genes which are silent (unexpressed) in the differentiated adult tissue are reactivated during cellular reprogramming (iPS) and cancer as well. Back in time, the first pluripotent cells were derived from teratocarcinoma (a germline tumor). When (explanted) grown in tissue culture, teratocarcinoma generated embryonic carcinoma cells. This happens due to reprogramming of cancer cell to a pluripotent state [62]. Therefore, it needs careful observation to limit the growth of iPS towards tumors/malignancy.

The success of iPS in clinical setting will be determined by safety, genomic integrity, programming efficiency, and so forth. The Rb-p53-p16 (INK4a) is a very important pathway for cell cycle progression, cell proliferation, and scenescence. At the molecular level, cancer of all kinds converges in p53 pathway. Due to mutation of Rb, p53 or p16 cell cycle progresses uncontrollably and untimely. p53 is a tumor suppressor gene and is dubbed as the guardian of the genome. Around $50-80 \%$ tumors of various kinds have p53 sporadic mutation [63]. The protein is quickly degraded (half life is around 30 mins). However, this protein is quickly activated (stabilized) during viral infection, untimed cell cycle progression, in DNA damage, and other stress [63]. After DNA damage the protein is phosphorylated by ATM/ATR kinases and stabilized. The protein upregulates the expression of the p21 gene that brakes the cell cycle progression from G1 to $\mathrm{S}$ phase. Among many genes, it also upregulates bax expression, a proapoptotic gene. During the cell cycle blockage, p53 assesses the degree/extent of DNA damage. If it is repairable, then it recruits/upregulates the DNA repair proteins and restores the integrity of the genome. But if the DNA damage is significant, then it directs the cells towards apoptosis. As ES and iPS cells also proliferate rapidly, the intact p53 pathway is an impediment of efficient reprogramming. Cell cycle and apoptosis act as a rate-limiting step during reprogramming. Anything that accelerates cell division, helps in faster reprogramming. It can be done if p53 is knocked down temporarily by p53 or p21 specific short hairpin (shRNA). This may facilitate faster reprogramming but also increases the chance of tumor development. Several labs have used temporary p53 knockdown for faster reprogramming, and then/later on 
restoring p53 pathway after iPS generation helps to guard against the development of any cancer [64].

Rem 2 is a suppressor of the $\mathrm{p} 53$ pathway and is expressed higher in hESC. Rem 2 works by accelerating the cell cycle and simultaneously protecting from apoptosis through an effect on cyclin D1. Rem 2 is a major player of hESC pluripotency and self-renewal. Rem 2 is as efficient as c-Myc and both enhance reprogramming efficiency by eightfold from human somatic cells to iPS.

\section{The Epigenetical Modification}

The Epigenetical modification has been widely observed in cancer, during embryogenesis and cellular reprogramming. During the mammalian development genomewide epigenetic reprogramming takes place in vivo but in vitro epigenetic reprogramming is not so efficient. That is why it takes a long time to reprogram. Epigenetic changes that modify DNA and DNA packaging protein (called histones) alters gene expression patterns and regulate cell identity [65]. To reprogram the cells to iPS we need to understand the cellular reprogramming process as well as epigenetic changes of the genome that follows the reprogramming steps. Epigenetics is defined as heritable changes in phenotype or gene expression caused by mechanisms other than changes in the underlying DNA sequence. The epigenetic mechanism to be considered as another dimension/language other than DNA. Reprogramming is regulated not only by $4 \mathrm{RFs}$ but also by epigenetic modification. Epigenetic modification can happen at the DNA level by DNA methylation of promoter and methylation/acetylation of proteins (histone) that wrap the DNA; gene may be expressed or silenced. The protein acetylation facilitates the RNA synthesis machinery to access the promoter. Tumor suppressor gene silencing has been reported in various cancers by epigenetic mechanism resulting in unrestricted cell growth. In case of SCNT aberrant DNA methylation (epigenetic) pattern has resulted in an inability to reach its pluripotent state during development into blastocyst in cattle [66]. In such cases, histone deacetylase inhibitor trichostatin A (TSA) treatment increases the efficiency of development to term of SCNT embryos [67]. The promoters of Oct $3 / 4$ and nanog are highly methylated and silent in starting fibroblast cells but demethylated and active in iPS cells. iPS efficiency can be increased by the treatment with epigenetic modification drugs. These may be DNA methyltransferase inhibitors like 5'-Azacytidine and RG108, histone deacetylase inhibitors such as valproic acid and TSA, and histone methyltransferase inhibitor BIX-01294. It was expected that iPS cells may have the same epigenetic modification as ES cells. Later it was identified that there are 71 differential methylation regions (DMRs) between ES cells and iPS cells [68]. iPS cells are not as equal as ES cells in some aspects and iPS cells have some memory about the tissue of origin. This was evidenced by comperative gene expression profiling of ES and iPS cells. Kang $\mathrm{H}$ and Roh $\mathrm{S}$ showed that although murine SCNT embryos activated to reprogram with or without TSA (a strong inhibitor of histone deacetylase), TSA (treated
$11 \mathrm{~h}$ ) showed higher blastocyst rate $(21.1 \%)$ compared to nontreated embryos (3.4\%). TSA-treated embryo showed decreased expression of histone deacetylase (both HDAC1 and HDAC2) and DNA methylation genes (DNMT3a and DNMT3b) where expression levels increased for expression of histone acetyltransferase (P300 and CBP) and pluripotency markers (OCT4 and NANOG). Thus chemicals like TSA can be used to compensate for the shortcomings of iPS [55]. Epigenetic changes happen when differentiation takes place from pluripotency and vice versa [69]. The iPS cells that fulfill pluripotent criteria may contain heterogeneous profiles that affect lineage-specific differentiation. Moreover, cells reprogrammed by iPS may contain a residual memory persistent from the original parental source as well as some remnants of the programming process itself which leads to biased potential to differentiate into tissues like cardiac tissues [70]. By enforced expression of TFs, it has shown that although distinct iPS colonies morphologically looks like ES cells, at the molecular level they are very heterogenous in expressing various stage-specific in differentiation potential. They found only one type of cells that represents true iPS cells and others are reprogramming intermediates. Expression of cellular markers like TRA-1-60, DNMT3B, and REX1 can be found in fully reprogrammed cells. Contrary to our understanding AP, SSEA-4, GDF3, hTERT, and NANOG are insufficient markers. Pluripotency is maintained by an open chromatic state of cells. It may be achieved in 2 ways (a) factors that keep chromatin open globally to facilitate the entry of transcription machineries to the gene promoters, and (b) factors that act locally to silence the lineage specific genes until the initiation of differentiation [71]. Bioengineered stem cells can be used for replacement tissue for a number of diseases and has shown therapeutic benefits upon transplantation in animal models [72]. Tursun et al. was able to convert various neuron types (glutamatergic, cholinergic, and GABAergic) by the ectopic expression of only one transcription factor in C. elegans. This was achieved by the removal of the histone chaperone LIN53 (homologous to human RbAp46/48) that works as histone remodeling and modifying complex. It can be mimicked by chemical inhibition of histone deacetylases [73].

\section{Molecular Mechanism of Reprogramming}

The detailed molecular mechanism of cellular reprogramming is not known yet. Several of the reprogramming factors work with protein-protein interaction. These genes bind with promoters of several genes. Epigenetic modification facilitates enzymes/proteins access to the promoter. There are 2 sets of genes with opposing functions: upregulation of genes that are involved in stemness like STAT3 and ZIC3 [74] and downregulation of genes that are responsible for differentiation like PAX6, ATBF1, and SUZ12 [75]. During reprogramming of MEF, induction of SSEA-1 and repression of Thy-1 gene are noticed at the early stage of reprogramming [76]. More late stage endogenous pluripotent markers OCT3/4 and SOX2 as well as telomerase are expressed that add telomere at the end of chromosomes. 
Fully reprogrammed cells are positive for pluripotent marker SSEA-4 and negative for TRA-1-60 and fibroblast marker, CD13. They are also supposed to have inactivated retroviral promoter. iPS has been produced from various source of adult cells like mouse liver [77], pancreatic beta cells [78]. Work on signaling pathway shows that modulation of Wnt/ $\beta$-catenin, MAPK/ERK, TGF- $\beta$, and PI3K/AKT signaling pathway increases the likelihood of somatic cell reprogramming [79].

Prigione and Adjaye 2010 have demonstrated that human ES and iPS both in the undifferentiated state and all stages of differentiation have similar mitochondrial properties which are distinct from those of fibroblasts. This was done by global transcriptional profiling and suggests that mitochondrial profile remains similar upon differentiation [80].

\section{Incomplete Programming}

Incomplete programming of mouse colony tends to express lineage-specific genes. CD13 markers are lost in the early stages of iPS followed by diminished expression of GFP and increased expression of SSEA4 and TRA-1-60. Hoechst staining is also diminished at the late stage of reprogramming as stem cells tend to pump out this nuclear staining dye. In summary, starting with CD13+ human fibroblasts, at the 1 st and 2nd stages of dedifferentiation, both have GFP+, Hoechst bright and TRA-1-60 negative. But at the 2nd stage SSEA-4 positive which is negative in the 1st stage. Stages 2 and 3, both have CD13-, SSEA4+; GFP is negative at stage 3 but positive at stage 2; TRA-1-60 is positive at stage 3 and negative at stage 2; Hoechst is dim in stage 3 but bright at stage 2. However, the above-mentioned markers are not always enough as cells expressing these markers sometimes fail to expand. Together with these markers and growth properties they help to identify the real iPS cell lines. The incompletely reprogrammed cells may self-renew due to the presence of $c-M y c$. Removal of $c-M y c$ from reprogramming factors has shown significantly decreased number of incompletely reprogrammed colonies in the mouse and human [10]. For that reason, Hanna et al. used a soluble wnt 3 a that promotes iPS regeneration in the absence of $c-M y c$. Wnt-3a conditioned media can reprogram MEF cells [36]. Wnt signalling results in inhibition of GSK-3 and stabilization of cytoplasmic $\beta$-catenin.

Li et al. has created iPSC from somatic cell by genetic transduction that remains homogeneous. They developed a method where both human and rat iPSCs (riPSCs) can be maintained by LIF and a cocktail of ALK5 inhibitor, GSK3 inhibitor, and MEK inhibitor [60].

\section{Determination of Cell Integrity after iPS}

Before using iPS for lineage-specific differentiation, the quality and genetic integrity of iPS cells are determined. Cells are karyotyped to rule out any chromosomal aberrations and cells should be confirmed as diploid, normal. Expression of exogenous pluripotency markers and nonintegration of LGNSO genes to host chromosome is determined by RT-PCR and standard Southern blotting, respectively. To examine the coexpression of Lin28, Nanog, Sox2, and Oct4, transfected cells are subjected to Western blot or immunofluorescence analysis. Pluripotency is determined in vitro and in vivo. EB formation is one of the hallmarks for in vitro differentiation of ES cells. Teratoma formation in vivo is a test for pluripotency. Spontaneous differentiation of MC-iPS cells into mesoderm, endoderm, and ectoderm lineages is detected by immunofluorescence and RT-PCR of genes that are specific for differentiation. To examine the in vivo developmental potential of human iPS cells generated through reprogramming, cells are injected into the dorsal flank of 6-week-old immune-compromised SCID-beige mice. After eight weeks, teratoma formation is evaluated.

\section{Conclusion}

Although iPS cells are easy to create in the lab ES cells are the gold standards. The ES cells are far more efficient in deriving other types of tissues than iPS. However, using ES cells is not easy due to ethical reasons. Since the establishment of iPS as an alternative to ESC, a new avenue has opened in stem cell research. Now cells can be autologously reprogramed to pluripotency for therapeutic applications. Any adult cells like skin cells, muscle cells, and adipose tissue cells have been used for reprogramming purposes. The original viral transduction method has evolved to reprogramming methods where reprogramming factors can be delivered without viral vectors thus genome remained unperturbed. This has been done by plasmid, protein, mRNA transfection, and minicircle nucleofection. Different cell types have different levels of reprogramming potency and different level of requirements of reprogramming factors. This may suggest that there is still undiscovered factors remained that are needed for efficient and quick generation of iPS. On the other hand, some cells express few factors abundantly than other kind of cells thus obviating the necessity of the reprogramming factor for that cell type. Teratoma formation from iPS is a drawback and needs careful screening of iPS that gives rise to teratoma in vivo. Direct reprogramming (iPS) is in nascent stage. Further research will facilitate the possibility of iPS in wide ranging of tissue engineering and regenerative medicine making the technology more available for therapeutic purposes.

\section{References}

[1] F. Nottebohm, "From bird song to neurogenesis," Scientific American, vol. 260, no. 2, pp. 74-79, 1989.

[2] J. B. Gurdon, "The developmental capacity of nuclei taken from intestinal epithelium cells of feeding tadpoles," Journal of Embryology and Experimental Morphology, vol. 10, pp. 622640, 1962.

[3] I. Wilmut, A. E. Schnieke, J. McWhir, A. J. Kind, and K. H. Campbell, "Viable offspring derived from fetal and adult mammalian cells," Nature, vol. 385, no. 6619, pp. 810-813, 1997.

[4] B. A. Reynolds and S. Weiss, "Generation of neurons and astrocytes from isolated cells of the adult mammalian central 
nervous system," Science, vol. 255, no. 5052, pp. 1707-1710, 1992.

[5] I. Gunaseeli, M. X. Doss, C. Antzelevitch, J. Hescheler, and A. Sachinidis, "Induced pluripotent stem cells as a model for accelerated patient- and disease-specific drug discovery," Current Medicinal Chemistry, vol. 17, no. 8, pp. 759-766, 2010.

[6] P. J. Fairchild, N. J. Robertson, S. Cartland, K. F. Nolan, and H. Waldmann, "Cell replacement therapy and the evasion of destructive immunity," Stem Cell Reviews, vol. 1, no. 2, pp. 159-168, 2005.

[7] K. Takahashi and S. Yamanaka, "Induction of pluripotent stem cells from mouse embryonic and adult fibroblast cultures by defined factors," Cell, vol. 126, no. 4, pp. 663-676, 2006.

[8] F. Jia, K. D. Wilson, N. Sun et al., "A nonviral minicircle vector for deriving human iPS cells," Nature Methods, vol. 7, no. 3, pp. 197-199, 2010.

[9] L. Warren, P. D. Manos, T. Ahfeldt et al., "Highly efficient reprogramming to pluripotency and directed differentiation of human cells with synthetic modified mRNA," Cell Stem Cell, vol. 7, no. 5, pp. 618-630, 2010.

[10] J. W. Han and Y. S. Yoon, "Induced pluripotent stem cells: emerging techniques for nuclear reprogramming," Antioxidants \& Redox Signaling, vol. 15, no. 7, pp. 1799-1820.

[11] C. Y. Fong, K. Gauthaman, and A. Bongso, "Teratomas from pluripotent stem cells: a clinical hurdle," Journal of Cellular Biochemistry, vol. 111, no. 4, pp. 769-781, 2010.

[12] N. Sun, N. J. Panetta, D. M. Gupta et al., "Feeder-free derivation of induced pluripotent stem cells from adult human adipose stem cells," Proceedings of the National Academy of Sciences of the United States of America, vol. 106, no. 37, pp. 15720-15725, 2009.

[13] S. G. Dubois, E. Z. Floyd, S. Zvonic et al., "Isolation of human adipose-derived stem cells from biopsies and liposuction specimens," Methods in Molecular Biology, vol. 449, pp. 69-79, 2008.

[14] G. R. Erickson, J. M. Gimble, D. M. Franklin, H. E. Rice, H. Awad, and F. Guilak, "Chondrogenic potential of adipose tissue-derived stromal cells in vitro and in vivo," Biochemical and Biophysical Research Communications, vol. 290, no. 2, pp. 763-769, 2002.

[15] M. J. Seo, S. Y. Suh, Y. C. Bae, and J. S. Jung, "Differentiation of human adipose stromal cells into hepatic lineage in vitro and in vivo," Biochemical and Biophysical Research Communications, vol. 328, no. 1, pp. 258-264, 2005.

[16] S. Rangappa, J. W. C. Entwistle, A. S. Wechsler, and J. Y. Kresh, "Cardiomyocyte-mediated contact programs human mesenchymal stem cells to express cardiogenic phenotype," Journal of Thoracic and Cardiovascular Surgery, vol. 126, no. 1, pp. 124-132, 2003.

[17] K. M. Safford, K. C. Hicok, S. D. Safford et al., "Neurogenic differentiation of murine and human adipose-derived stromal cells," Biochemical and Biophysical Research Communications, vol. 294, no. 2, pp. 371-379, 2002.

[18] T. Scholz, S. Satyanarayan, S. Dhar, and G. R. D. Evans, "Correlation of rapid phenotypic changes and insulin production of differentiated human adipose tissue-derived stem cells," Annals of Plastic Surgery, vol. 63, no. 4, pp. 436-440, 2009.

[19] B. Cousin, M. André, E. Arnaud, L. Pénicaud, and L. Casteilla, "Reconstitution of lethally irradiated mice by cells isolated from adipose tissue," Biochemical and Biophysical Research Communications, vol. 301, no. 4, pp. 1016-1022, 2003.
[20] T. Matsui, D. Leung, H. Miyashita et al., "Proviral silencing in embryonic stem cells requires the histone methyltransferase ESET," Nature, vol. 464, no. 7290, pp. 927-931, 2010.

[21] K. Takahashi, K. Tanabe, M. Ohnuki et al., "Induction of pluripotent stem cells from adult human fibroblasts by defined factors," Cell, vol. 131, no. 5, pp. 861-872, 2007.

[22] K. Okita, T. Ichisaka, and S. Yamanaka, "Generation of germline-competent induced pluripotent stem cells," Nature, vol. 448, no. 7151, pp. 313-317, 2007.

[23] C. A. Sommer, A. G. Sommer, T. A. Longmire et al., "Excision of reprogramming transgenes improves the differentiation potential of iPS cells generated with a single excisable vector," Stem Cells, vol. 28, no. 1, pp. 64-74, 2010.

[24] T. S. Mikkelsen, J. Hanna, X. Zhang et al., "Dissecting direct reprogramming through integrative genomic analysis," Nature, vol. 454, no. 7200, pp. 49-55, 2008.

[25] G. J. Hannon, "RNA interference," Nature, vol. 418, no. 6894, pp. 244-251, 2002.

[26] E. Yakubov, G. Rechavi, S. Rozenblatt, and D. Givol, "Reprogramming of human fibroblasts to pluripotent stem cells using mRNA of four transcription factors," Biochemical and Biophysical Research Communications, vol. 394, no. 1, pp. 189193, 2010.

[27] P. A. Zuk, M. Zhu, P. Ashjian et al., "Human adipose tissue is a source of multipotent stem cells," Molecular Biology of the Cell, vol. 13, no. 12, pp. 4279-4295, 2002.

[28] C. Bichsel, D. K. Neeld, T. Hamazaki et al., "Bacterial delivery of nuclear proteins into pluripotent and differentiated cells," PLoS ONE, vol. 6, no. 1, Article ID e16465, 2011.

[29] C. Voelkel, M. Galla, T. Maetzig et al., "Protein transduction from retroviral Gag precursors," Proceedings of the National Academy of Sciences of the United States of America, vol. 107, no. 17, pp. 7805-7810, 2010.

[30] J. Han, P. Yuan, H. Yang et al., "Tbx3 improves the germ-line competency of induced pluripotent stem cells," Nature, vol. 463, no. 7284, pp. 1096-1100, 2010.

[31] S. Y. Tsai, C. Clavel, S. Kim et al., "Oct4 and Klf4 reprogram dermal papilla cells into induced pluripotent stem cells," Stem Cells, vol. 28, no. 2, pp. 221-228, 2010.

[32] J. H. Ng and H. H. Ng, "LincRNAs join the pluripotency alliance," Nature Genetics, vol. 42, no. 12, pp. 1035-1036, 2010.

[33] Y. Jeong and D. J. Mangelsdorf, "Nuclear receptor regulation of stemness and stem cell differentiation," Experimental and Molecular Medicine, vol. 41, no. 8, pp. 525-537, 2009.

[34] L. Huang, N. Fan, J. Cai et al., "Establishment of a porcine Oct-4 promoter-driven EGFP reporter system for monitoring pluripotency of porcine stem cells," Cellular Reprogramming, vol. 13, no. 2, pp. 93-98, 2011.

[35] S. Sugii, Y. Kida, W. T. Berggren, and R. M. Evans, "Feederdependent and feeder-independent iPS cell derivation from human and mouse adipose stem cells," Nature Protocols, vol. 6, no. 3, pp. 346-358, 2011.

[36] J. Hanna, S. Markoulaki, P. Schorderet et al., "Direct reprogramming of terminally differentiated mature B lymphocytes to pluripotency," Cell, vol. 133, no. 2, pp. 250-264, 2008.

[37] E. P. Papapetrou, M. J. Tomishima, S. M. Chambers et al., "Stoichiometric and temporal requirements of Oct4, Sox2, Klf4, and c-Myc expression for efficient human iPSC induction and differentiation," Proceedings of the National Academy of Sciences of the United States of America, vol. 106, no. 31, pp. 12759-12764, 2009. 
[38] A. Martinez-Fernandez, T. J. Nelson, and A. Terzic, "Nuclear reprogramming strategy modulates differentiation potential of induced pluripotent stem cells," Journal of Cardiovascular Translational Research, vol. 4, no. 2, pp. 131-137, 2011.

[39] N. Maki, J. Martinson, O. Nishimura et al., "Expression profiles during dedifferentiation in newt lens regeneration revealed by expressed sequence tags," Molecular Vision, vol. 16, pp. 72-78, 2010.

[40] M. A. Esteban, T. Wang, B. Qin et al., "Vitamin C enhances the generation of mouse and human induced pluripotent stem cells," Cell Stem Cell, vol. 6, no. 1, pp. 71-79, 2010.

[41] T. Graf and T. Enver, "Forcing cells to change lineages," Nature, vol. 462, no. 7273, pp. 587-594, 2009.

[42] X. Yuan, W. Li, and S. Ding, "Small molecules in cellular reprogramming and differentiation," Progress in Drug Research, vol. 67, pp. 253-266, 2011.

[43] L. Anastasia, G. Pelissero, B. Venerando, and G. Tettamanti, "Cell reprogramming: expectations and challenges for chemistry in stem cell biology and regenerative medicine," Cell Death and Differentiation, vol. 17, no. 8, pp. 1230-1237, 2010.

[44] W. Li, H. Zhou, R. Abujarour et al., "Generation of humaninduced pluripotent stem cells in the absence of exogenous Sox2," Stem Cells, vol. 27, no. 12, pp. 2992-3000, 2009.

[45] K. I. Nagai, H. Ishii, N. Miyoshi et al., "Long-term culture following ES-like gene-induced reprogramming elicits an aggressive phenotype in mutated cholangiocellular carcinoma cells," Biochemical and Biophysical Research Communications, vol. 395, no. 2, pp. 258-263, 2010.

[46] J. T. Do and H. R. Schöler, "Cell fusion-induced reprogramming," Methods in Molecular Biology, vol. 636, pp. 179-190, 2010.

[47] X. S. Yue, M. Fujishiro, M. Toyoda, T. Akaike, and Y. Ito, "Reprogramming of somatic cells induced by fusion of embryonic stem cells using hemagglutinating virus of Japan envelope (HVJ-E)," Biochemical and Biophysical Research Communications, vol. 394, no. 4, pp. 1053-1057, 2010.

[48] A. E. Powell, E. C. Anderson, P. S. Davies et al., "Fusion between intestinal epithelial cells and macrophages in a cancer context results in nuclear reprogramming," Cancer Research, vol. 71, no. 4, pp. 1497-1505, 2011.

[49] R. S. Maser and R. A. DePinho, "Connecting chromosomes, crisis, and cancer," Science, vol. 297, no. 5581, pp. 565-569, 2002.

[50] R. M. Marión and M. A. Blasco, "Telomeres and telomerase in adult stem cells and pluripotent embryonic stem cells," Advances in Experimental Medicine and Biology, vol. 695, pp. $118-131,2010$.

[51] S. T. Suhr, E. A. Chang, R. M. Rodriguez et al., "Telomere dynamics in human cells reprogrammed to pluripotency," PLoS ONE, vol. 4, no. 12, Article ID e8124, 2009.

[52] D. Eberhard, K. O’Neill, Z. D. Burke, and D. Tosh, "In vitro reprogramming of pancreatic cells to hepatocytes," Methods in Molecular Biology, vol. 636, pp. 285-292, 2010.

[53] C. Cobaleda, "Reprogramming of B cells," Methods in Molecular Biology, vol. 636, pp. 233-250, 2010.

[54] A. B. Mak, Z. Ni, J. A. Hewel et al., "A lentiviral functional proteomics approach identifies chromatin remodeling complexes important for the induction of pluripotency," Molecular and Cellular Proteomics, vol. 9, no. 5, pp. 811-823, 2010.

[55] H. Kang and S. Roh, "Extended exposure to trichostatin A after activation alters the expression of genes important for early development in nuclear transfer murine embryos,"
Journal of Veterinary Medical Science, vol. 73, no. 5, pp. 623631, 2011.

[56] D. Huangfu, K. Osafune, R. Maehr et al., "Induction of pluripotent stem cells from primary human fibroblasts with only Oct4 and Sox2," Nature Biotechnology, vol. 26, no. 11, pp. 1269-1275, 2008.

[57] N. Sato, L. Meijer, L. Skaltsounis, P. Greengard, and A. H. Brivanlou, "Maintenance of pluripotency in human and mouse embryonic stem cells through activation of Wnt signaling by a pharmacological GSK-3-specific inhibitor," Nature Medicine, vol. 10, no. 1, pp. 55-63, 2004.

[58] H. Umehara, T. Kimura, S. Ohtsuka et al., "Efficient derivation of embryonic stem cells by inhibition of glycogen synthase kinase-3," Stem Cells, vol. 25, no. 11, pp. 2705-2711, 2007.

[59] H. K. Bone, T. Damiano, S. Bartlett et al., "Involvement of GSK-3 in regulation of murine embryonic stem cell self-renewal revealed by a series of bisindolylmaleimides," Chemistry and Biology, vol. 16, no. 1, pp. 15-27, 2009.

[60] W. Li and S. Ding, "Generation of novel rat and human pluripotent stem cells by reprogramming and chemical approaches," Methods in Molecular Biology, vol. 636, pp. 293 300, 2010.

[61] B. M. Simon, C. E. Murdoch, and C. T. Scott, "Pluripotent patents make prime time: an analysis of the emerging landscape," Nature Biotechnology, vol. 28, no. 6, pp. 557-559, 2010.

[62] B. L. M. Hogan, "Changes in the behaviour of teratocarcinoma cells cultivated in vitro," Nature, vol. 263, no. 5573, pp. 136137, 1976.

[63] A. J. Levine, C. A. Finlay, and P. W. Hinds, "P53 is a tumor suppressor gene,” Cell, vol. 116, supplement 2, pp. S67-S69, 2004.

[64] A. Banito and J. Gil, "Induced pluripotent stem cells and senescence: learning the biology to improve the technology," EMBO Reports, vol. 11, no. 5, pp. 353-359, 2010.

[65] A. D. Goldberg, C. D. Allis, and E. Bernstein, "Epigenetics: a landscape takes shape," Cell, vol. 128, no. 4, pp. 635-638, 2007.

[66] J. Suzuki Jr., J. Therrien, F. Filion et al., "Loss of methylation at H19 DMD is associated with biallelic expression and reduced development in cattle derived by somatic cell nuclear transfer," Biology of Reproduction, vol. 84, no. 5, pp. 947-956, 2011.

[67] T. Hai, J. Hao, L. Wang, A. Jouneau, and Q. Zhou, "Pluripotency maintenance in mouse somatic cell nuclear transfer embryos and its improvement by treatment with the histone deacetylase inhibitor TSA," Cellular Reprogramming, vol. 13, no. 1, pp. 47-56, 2011.

[68] A. Doi, I. H. Park, B. Wen et al., "Differential methylation of tissue-and cancer-specific CpG island shores distinguishes human induced pluripotent stem cells, embryonic stem cells and fibroblasts," Nature Genetics, vol. 41, no. 12, pp. 1350$1353,2009$.

[69] A. Martinez-Fernandez, T. J. Nelson, and A. Terzic, "Nuclear reprogramming and pluripotency," Nature, vol. 441, pp. 10611067, 2006.

[70] A. Martinez-Fernandez, T. J. Nelson, Y. Ikeda, and A. Terzic, "c-MYC-independent nuclear reprogramming favors cardiogenic potential of induced pluripotent stem cells," Journal of Cardiovascular Translational Research, vol. 3, no. 1, pp. 13-23, 2010.

[71] A. Gaspar-Maia, A. Alajem, E. Meshorer, and M. RamalhoSantos, "Open chromatin in pluripotency and reprogramming," Nature Reviews Molecular Cell Biology, vol. 12, no. 1, pp. 36-47, 2011. 
[72] T. J. Nelson, A. Martinez-Fernandez, S. Yamada, Y. Ikeda, C. Perez-Terzic, and A. Terzic, "Induced pluripotent stem cells: advances to applications," Stem Cells and Cloning: Advances and Applications, vol. 3, no. 1, pp. 29-37, 2010.

[73] B. Tursun, T. Patel, P. Kratsios, and O. Hobert, "Direct conversion of C. elegans germ cells into specific neuron types," Science, vol. 331, no. 6015, pp. 304-308, 2010.

[74] L. A. Boyer, I. L. Tong, M. F. Cole et al., "Core transcriptional regulatory circuitry in human embryonic stem cells," Cell, vol. 122, no. 6, pp. 947-956, 2005.

[75] L. A. Boyer, K. Plath, J. Zeitlinger et al., "Polycomb complexes repress developmental regulators in murine embryonic stem cells," Nature, vol. 441, no. 7091, pp. 349-353, 2006.

[76] M. Stadtfeld, N. Maherali, D. T. Breault, and K. Hochedlinger, "Defining molecular cornerstones during fibroblast to iPS cell reprogramming in mouse," Cell Stem Cell, vol. 2, no. 3, pp. 230-240, 2008.

[77] T. Aoi, K. Yae, M. Nakagawa et al., "Generation of pluripotent stem cells from adult mouse liver and stomach cells," Science, vol. 321, no. 5889, pp. 699-702, 2008.

[78] M. Stadtfeld, K. Brennand, and K. Hochedlinger, "Reprogramming of pancreatic $\beta$ cells into induced pluripotent stem cells," Current Biology, vol. 18, no. 12, pp. 890-894, 2008.

[79] D. Sanges and M.-P. Cosma, "Reprogramming cell fate to pluripotency: the decision-making signalling pathways," The International Journal of Developmental Biology, vol. 54, no. 1112, pp. 1575-1587, 2011.

[80] A. Prigione and J. Adjaye, "Modulation of mitochondrial biogenesis and bioenergetic metabolism upon in vitro and in vivo differentiation of human ES and iPS cells," The International Journal of Developmental Biology, vol. 54, no. 1112, pp. 1729-1741, 2010. 

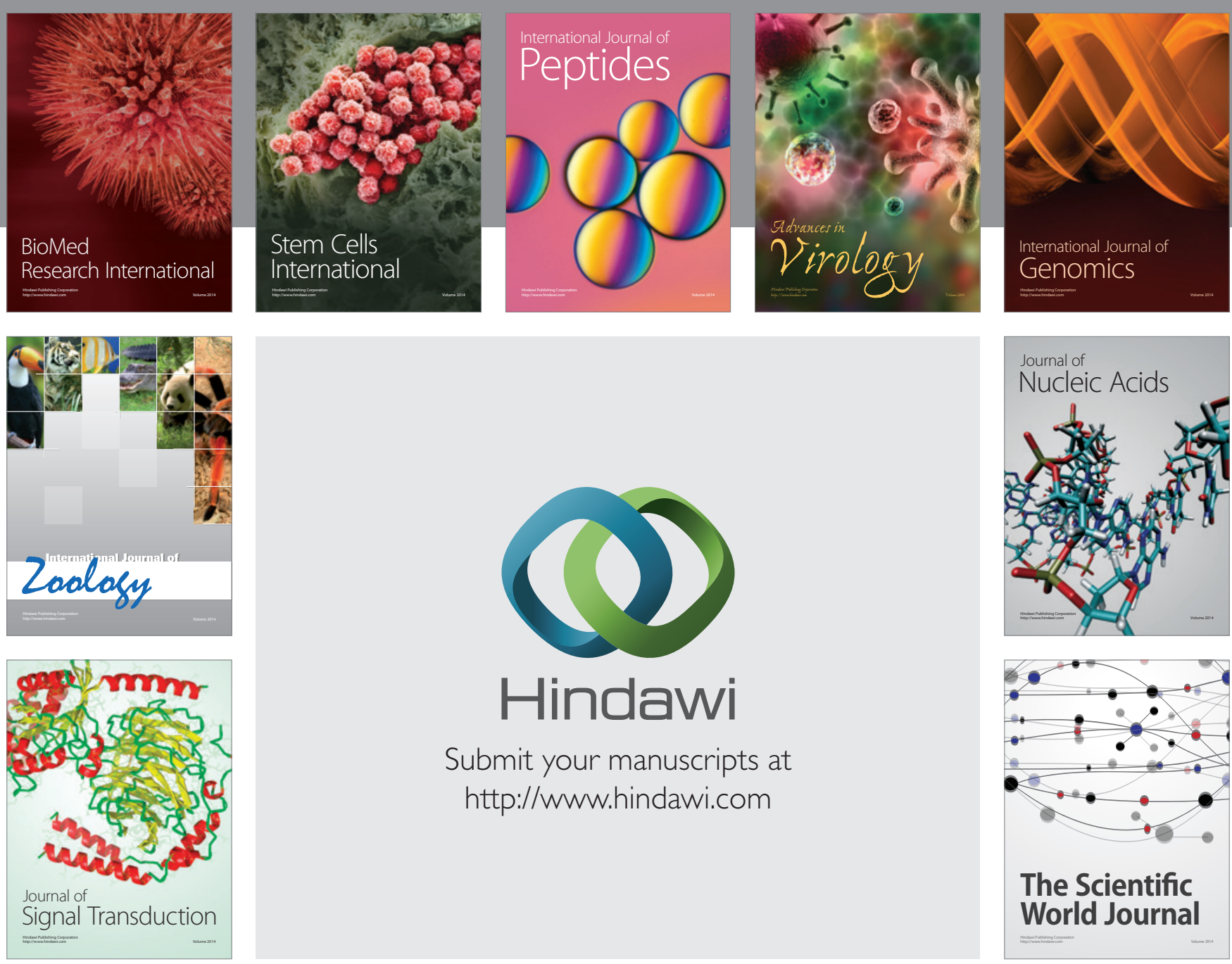

Submit your manuscripts at

http://www.hindawi.com
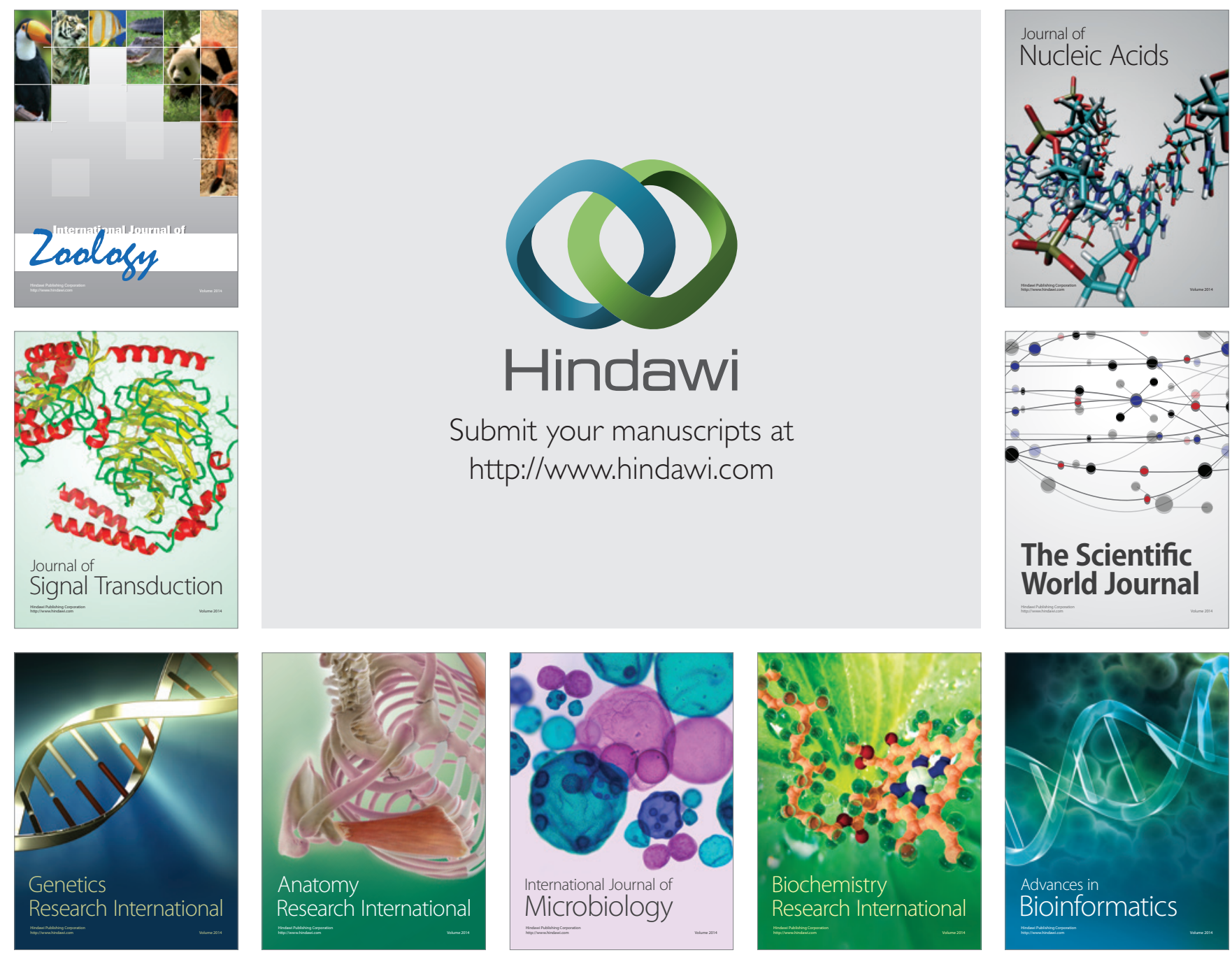

The Scientific World Journal
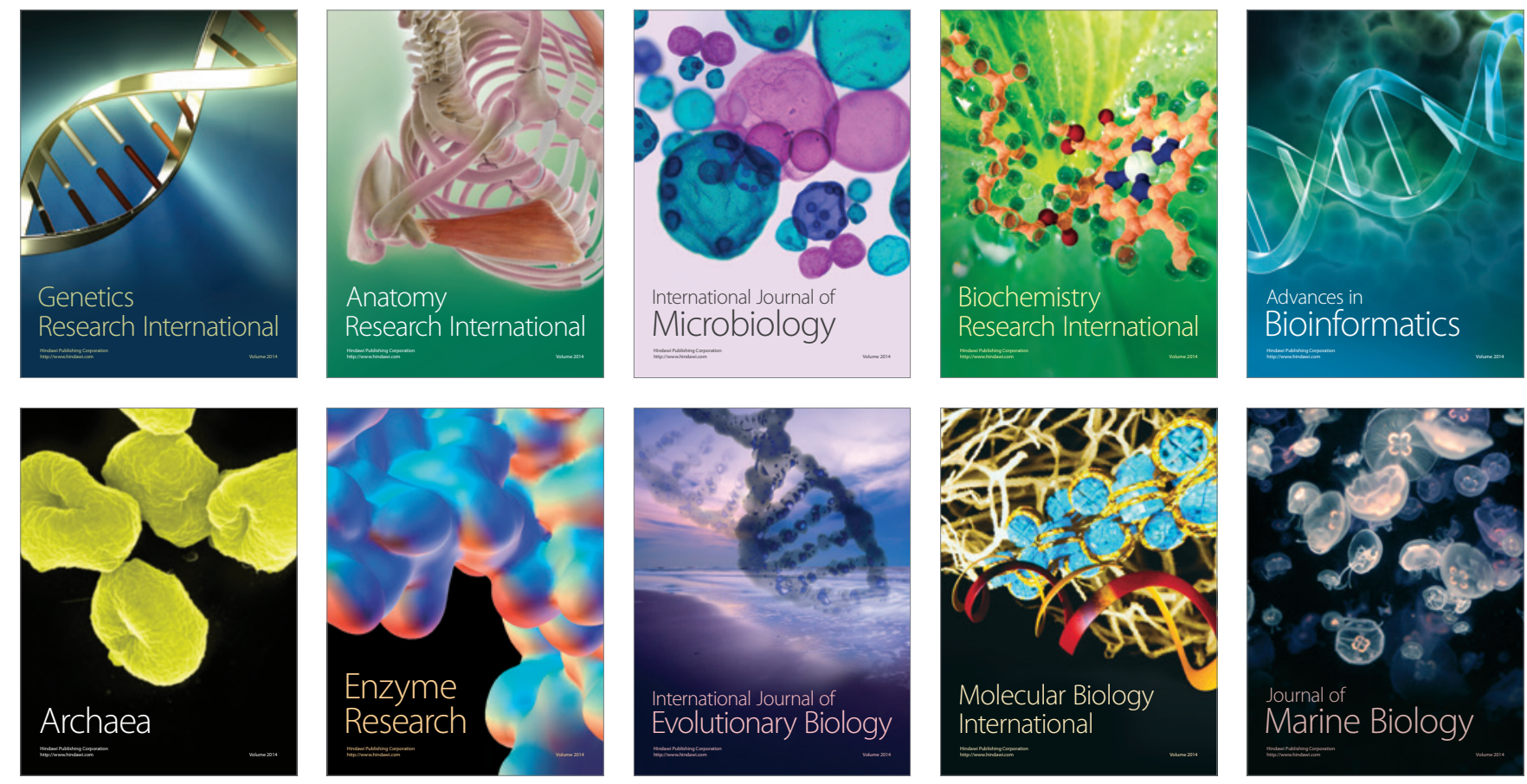